neonatal central DI. Six cases were preterm with intracranial hemorrhage and the other two patients were congenital toxoplasmosis one of which was preterm. All of the cases received oral desmopres$\sin$ at a dose of $10 \mathrm{mcg} / \mathrm{kg} /$ day. And then oral desmopressin dosage was adjusted according to the serum sodium and urine output. All cases were treated successfully with oral DDAVP. Three cases who have intracranial hemorrhage died due to other preterm complication. According to our case series, oral DDAVP is an applicable, safe and effective form of DDAVP.

\section{INTER-HOSPITAL TRANSPORT OF NEONATES IN THE SOUTH OF VIETNAM - RELATIONS TO PROGNOSIS AND OUTCOME}

doi:10.1136/archdischild-2012-302724.1322

${ }^{1} \mathrm{JIM}$ Andersen, 'MM Andersen, ${ }^{2} \mathrm{FK}$ Pedersen. 'University of Copenhagen; ${ }^{2}$ Pediatric Clinic, Juliane Marie Centeret, Rigshospitalet, Copenhagen, Denmark

Background and Aim To improve neonatal conditions in the South of Vietnam through mapping the conditions during interhospital transfer, revealing avoidable factors and unmasking cultural differences allowing action towards improvements.

Method A prospective and analytical cohort study was conducted at Pediatric Hospital \#1 in HCM City, Vietnam. Data was collected through standardized questionnaires and during a set timeframe in a one-month period. Infants $<28$ days or weighing $<2000 \mathrm{~g}$, transferred from the south-half of Vietnam were included giving a total of 103 neonates representing 96 transfers.

Results A transfer $>120$ min increased the risk of admission $>8$ days in total $(p=.027)$ and in NICU $(p=.001)$. The infants most vulnerable to transfer were more frequently transferred by an ambulance equipped and escorted to handle emergency than the least vulnerable $(\mathrm{p}=.003)$. However, the transfers of intermediate quality (51.5\%) had significantly worse outcomes ( $p=.007) .28 .2 \%$ of the ambulances were adequately equipped to handle emergencies and none had monitoring equipment for neonates. All neonates were escorted by health personnel, though none had transport training and only $32 \%$ had appropriate qualifications. Initiated treatment was continued, but no new initiatives were taken.

Conclusion The inter-hospital transport is of good standard, all transfers being by ambulance, with medical accompaniment and some pre-assessment made. A long transfer still worsens the outcome of the neonates significantly. However, our study reveals factors, which can be improved prior to, during and following transfer. In order to succeed with new approaches, the cultural aspects must be acknowledged.

\section{IMPACT OF THE NEAR TERM INFANTS ON NICU BED UTILIZATION}

doi:10.1136/archdischild-2012-302724.1323

EH Abdulrahman, MM Abou Al-Seoud. Neonatal Division-Paediatric Department, King Fahad Armed Forces Hospital, Jeddah, Saudi Arabia

Background Near term infants are prone to more medical problems than full term infants requiring more neonatal intensive careunit (NICU) admissions

Aim Study the impact of near term infants on the utilization of NICU beds.

Methods Retrospective collection of hospital newborn electronic database from January 2007 - December 2011.

Two groups were identified group 1 infants 37 weeks and above and group 2 near term infants 34 weeks- $36+6$ weeks. The following demographics collected, Birth weight (BWT), Gestational age (GA), gender, length of stay (LOS) and NICU admission.

Results The number of newborns delivered during the study period were41089. Near term infants were 3722 (9\%). Mean birth weight was $3.1 \pm 0.458 \mathrm{~kg}$ for group 1 and $2.42 \pm 497 \mathrm{~kg}$ for group 2 (p 0.0001). 5835 (14\%)infants required NICU admissions at birth in group 1 and 1025(28\%) in group 2 (p 0.09). LOS was calculated a mean of $2.58 \pm 3.33$ days in group 1 and $8.5 \pm 15.58$ days in group 2 (p.0001).

Using multiple regression analysis birth weight and male gender were predictors for prolonged hospital LOS and the need for NICU admission in both groups.

Conclusion In our population near term infants were more likely to utilize NICU beds for significantly longer LOS compared to full term which increases costs and limit availability of NICU beds in our community.

\section{CLINICAL PROFILE AND OUTCOME OF NEWBORNS WITH ACUTE KIDNEY INJURY IN A LEVEL 3 NEONATAL UNIT IN WESTERN INDIA}

doi:10.1136/archdischild-2012-302724.1324

AR Kungwani, AS Nimbalkar, AR Sethi, DV Patel, SM Nimbalkar. Department of Pediatrics, Pramukhswami Medical College, Anand, India

\section{Background and Aims Our study aims are}

1. To study the clinical profile and associated conditions in newborns with Acute Kidney Injury (AKI) in our Level 3 Neonatal ICU.

2. To evaluate the incidence and outcome of newborns with AKI.

3. To study risk factors associated with AKI in our newborns.

Methods AKI was defined as Serum Creatinine $>1.5 \mathrm{mg} / \mathrm{dl}$. Data about neonates admitted over period of three years were studied from a Level 3 Neonatal Intensive Care Unit in Western India. Parameters associated with AKI were evaluated and descriptive analysis was done.

Results Out of 1745 patients, 74 (Male-61, Female-13) patients had AKI. The incidence of AKI was $4.24 \%$ of admitted newborns, and $80 \%$ of the babies developed AKI within first week. Incidence of AKI in outborn babies (5.83\%) is higher than inborn babies $(2.44 \%)$ and outborns comprised $72.97 \%$ of all AKIs (54/74). Mean Birth weight of neonates was $2.46 \pm 0.55 \mathrm{~kg}$ with $47.30 \%$ being LBWI. Factors associated with AKI were sepsis (91.9\%), shock(64.9\%), requirement of ventilation $(62.2 \%)$, Perinatal asphyxia (36.5\%), resuscitation $(40.5 \%)$ and requirement of intubation at birth (23\%). Mean Serum Creatinine was $2.87 \pm 1.81 \mathrm{mg} / \mathrm{dl}(1.51$ to 10.05$)$. Mean age at diagnosis was $5.76 \pm 6.64$ days(1 to 41 ). The mortality was $20 \%$ and $51.6 \%$ patients went DAMA (Discharge Against Medical Advice).

Conclusions Mortality in patients with AKI is very high. Most AKI occurs in the first week of life and factors associated with AKI are easily recognizable and should prompt early referral of neonates.

\section{5 \\ INTRA-SUBJECT VARIABILITY ON 3T MRI IMAGING OF NEONATAL HIPS}

doi:10.1136/archdischild-2012-302724.1325

${ }^{1} \mathrm{H}$ Hill, ${ }^{2} \mathrm{~A}$ Murphy, ${ }^{3} \mathrm{E}$ Jacqz-Aigrain, ${ }^{4} \mathrm{LJ}$ Abernethy, ${ }^{1} \mathrm{MA}$ Turner, TINN (Treat INfections in Neonates). 'Women's and Chidren's Health, University of Liverpool; ${ }^{2}$ Neonatal Unit, Liverpool Women's NHS Foundation Trust, Liverpool, UK; ${ }^{3}$ Paediatric Pharmacology and Pharmacogenetics, Robert Debré Hospital - APHP, Paris, France; ${ }^{4}$ Clinical Radiology, Alder Hey NHS Foundation Trust, Liverpool, UK

Background MRI may have utility for examining neonatal hips. To date, MRI measurements of hip dimensions have only been characterized on post-mortem imaging. The aim of this study was to compare measurements made on left and right neonatal hips.

Methods Neonatal hips were imaged at 3T. Acetabular width and depth were measured using methods described by Whitby 